\title{
Perturbation theory for electrical resistivity of liquid transition metals
}

\author{
V.T.Shvets, S.Savenko, S.Datsko \\ Odesa State Academy of Refrigeration, \\ 1/3 Dvorianskaia Str., 65026 Odesa, Ukraine
}

Received April 10, 2002

\begin{abstract}
Nearly free electron model is used for the $s$-electron subsystem of liquid transition metals. $d$-electrons are considered to be bound and serve as an additional scattering factor. Model Hamiltonian of the electron subsystem contains two small parameters: pseudopotential of $s$-electron-ion interaction, and $s-d$ hybridization potential. The linear response theory of Kubo and the method of two-time retarded Green functions are used to investigate the conductivity of the system. The perturbation series by two small system parameters are first derived for the electrical resistivity of liquid transition metals. To introduce the electron-electron interaction, a random phase approach is employed. The analysis of the second and the third terms of perturbation series is given. Their contribution to the electrical resistivity of all liquid transition metals is estimated.
\end{abstract}

Key words: electroresistivity, metal

PACS: 72.14.E, 72.15.C

\section{Introduction}

The theory of electron transport phenomena in the liquid simple metals dates back from the Ziman's work, where the inverse relaxation time was calculated in the second order by pseudopotential of electron-ion interaction. In succeeding years the perturbation theory was developed and the numerical calculations of the terms up to the third order were accomplished (the detailed list of the corresponding papers is available in the work [2]). At present, the theory of the electron transport phenomena in the liquid simple metals has taken its complete shape. The investigation of transition metals started much later and much of the work done is confined within two various trends. The first one goes back to the work [3] and represents the generalization of the pseudopotential theory to the case of transition metals. From the formal point of view it comes to the substitution of the single-particle $T$-matrix for the formfactor of pseudopotential. Further on, the resistivity is calculated in the second order by $T$-matrix $[4,5]$. Another trend is connected with Mott 
$[6,9,10]$, and is based on the idea that the basic factor causing the scattering of conduction electrons is hybridization of $s$ - and $d$ - states of the electron subsystem of a metal. At that $d$-electrons are considered to be localized and the effect is described using a hybridization potential. The formula proposed suits the second order of the perturbation theory by hybridization potential. Subsequently, no attempts were made neither of combining these two approaches, nor of constructing the perturbation series in the framework of each one. Unexpectedly, the progress was made in quite different direction. The starting point of these investigations was the tightbinding approximation [7-10], unlike all the previous cases where the nearly free electron model was employed. It was found that the development of perturbation series is more convenient for the metals with relatively high resistance (more than $200 \mu \Omega \cdot \mathrm{cm}$ ) are put under consideration. Since nearly half of the transition metals do not meet these conditions, the problem remains rather acute for transition metals with a relatively small resistivity. The purpose of the current paper is to build the perturbation series for resistivity of liquid transition metals using two small theory parameters: pseudopotential and the hybridization potential, with the subsequent analysis of the third order term.

\section{Hamiltonian}

Let us choose the model Hamiltonian of the electron subsystem of transition liquid metal in the form:

$$
H=H_{0}+H_{i s}+H_{s d}
$$

where

$$
H_{0}=\sum_{k} \varepsilon_{\mathbf{k}} a_{\mathbf{k}}^{+} a_{\mathbf{k}}+\sum_{n} \varepsilon_{n} b_{n}^{+} b_{n}
$$

is the Hamiltonian of non-interacting subsystems of $s$ - and $d$ - electrons;

$$
\varepsilon_{\mathbf{k}}=\frac{\hbar^{2} k^{2}}{2 m}
$$

is the energy of a free $s$-electron, $\varepsilon_{d}$ is the energy of a bound $d$-electron, $a_{\mathbf{k}}^{+}$and $a_{\mathbf{k}}$ are the operators of creation and annihilation of $s$-electrons in the state with quantum number $\mathbf{k}, b_{n}^{+}$and $b_{n}$ are the operators of creation and annihilation of $d$-electrons in the state with the set of quantum numbers $n$, which includes the ion number as well;

$$
H_{i s}=V^{-1} \sum_{\mathbf{q}} w(q) \rho^{i}(\mathbf{q}) \rho^{e}(-\mathbf{q})
$$

is the Hamiltonian of $s$-electron-ion interaction. It's assumed here that this interaction can be described using pseudopotential, just like in the case of a simple metal. In the expression above $w(q)$ is the screened formfactor of the local model pseudopotential,

$$
\rho^{i}(\mathbf{q})=\sum_{n} \exp \left(-\mathrm{iq} \mathbf{R}_{n}\right)
$$


is the Fourier transform of the ion density, $\mathbf{R}_{n}$ is the radius-vector of the $n$-th ion and

$$
\rho^{e}(\mathbf{q})=\sum_{\mathbf{k}} a_{\mathbf{k}}^{+} a_{\mathbf{k}+\mathbf{q}}
$$

is the Fourier transform of $s$-electron density operator. The last term of expression (1)

$$
H_{s d}=V^{-1 / 2} \sum_{\mathbf{k}, n}\left[\Delta_{\mathbf{k} n} a_{\mathbf{k}}^{+} b_{n}+\Delta_{n \mathbf{k}} b_{n}^{+} a_{\mathbf{k}}\right]
$$

is the Hamiltonian of $s$ - $d$ hybridization, where $V$ is the volume of the system and

$$
\begin{aligned}
\Delta_{\mathbf{k} n} & =\Delta_{k} \exp \left(-\mathrm{ik} \mathbf{R}_{n}\right) \\
\Delta_{n \mathbf{k}} & =\Delta_{k} \exp \left(\mathrm{ikR}_{n}\right)
\end{aligned}
$$

are the matrix elements of hybridization potential, $\Delta_{k}$ is the formfactor.

The present Hamiltonian doesn't include the electron-electron interaction explicitly. However, it is accounted implicitly via the screening of electron-ion interaction. Here we also neglected the exchange of $d$-electrons between ions, considering that this effect gains importance only in the metals with relatively high resistivity.

\section{Conductance coefficient}

According to the Kubo's linear response theory we can write the conductivity coefficient $\sigma$ in the form

$$
\sigma=-\frac{1}{3 V k_{\mathrm{B}} T} \operatorname{Im}\langle\langle\mathbf{I}(t) \mathbf{I}(0)\rangle\rangle_{0}
$$

In the right part of this equation there is a zero component of the two-time retarded Green function, constructed from the operators of electric current

$$
\langle\langle\mathbf{I}(t) \mathbf{I}(0)\rangle\rangle=\frac{1}{\mathrm{i} \hbar}\langle\mathbf{I}(t) \mathbf{I}(0)\rangle,
$$

the angular brackets have the meaning of the grand canonical ensemble average. Assuming that the electric current is fully determined by $s$-electrons, we can write the current operator as

$$
\mathbf{I}(t)=\frac{e \hbar}{m} \sum_{\mathbf{k}} \mathbf{k} a_{\mathbf{k}}^{+}(t) a_{\mathbf{k}}(t) .
$$

Thus, to obtain the conductivity coefficient we should perform calculations of the following Green function: $\left\langle\left\langle a_{\mathbf{k}}^{+}(t) a_{\mathbf{k}}(t) \mathbf{I}(0)\right\rangle\right\rangle$. Henceforth, to save place, we will use the shorthand $\left\langle\left\langle a_{\mathbf{k}}^{+} a_{\mathbf{k}}\right\rangle\right\rangle$ for this expression. 


\section{The motion equations}

In contrast to amorphous alloys at a low temperature, the influence of the dynamics of ion subsystem is inessential for the liquid metals. So, it is possible to regard the ion subsystem as a static one. We will consider it to be static as well that allows us to use only the Hamiltonian of the electron subsystem while constructing the motion equations. The Fourier transform of the first equation in the infinite set of dependent equations will look as follows

$$
\begin{aligned}
\hbar \omega\left\langle\left\langle a_{\mathbf{k}}^{+} a_{\mathbf{k}}\right\rangle\right\rangle_{\omega}= & \left\langle a_{\mathbf{k}}^{+} a_{\mathbf{k}}\right\rangle+\sum_{\mathbf{q}}\left[\left\langle\left\langle a_{\mathbf{k}}^{+} W(\mathbf{q}) a_{\mathbf{k}-\mathbf{q}}\right\rangle\right\rangle_{\omega}-\left\langle\left\langle a_{\mathbf{k}+\mathbf{q}}^{+} W(\mathbf{q}) a_{\mathbf{k}}\right\rangle\right\rangle_{\omega}\right] \\
& +\sum_{\mathbf{k}, n}\left[\left\langle\left\langle a_{\mathbf{k}}^{+} \Delta_{\mathbf{k} n} b_{n}\right\rangle\right\rangle_{\omega}-\left\langle\left\langle b_{n}^{+} \Delta_{n \mathbf{k}} a_{\mathbf{k}}\right\rangle\right\rangle_{\omega}\right] .
\end{aligned}
$$

Here we have denoted

$$
W(\mathbf{q})=V^{-1} w(q) \rho^{i}(\mathbf{q}) .
$$

For each of the new Green functions, the motion equation should be constructed in turn. These four equations will take the form

$$
\begin{aligned}
\left(\varepsilon_{\mathbf{k}}-\right. & \left.\varepsilon_{\mathbf{k}-\mathbf{q}}+\hbar \omega\right)\left\langle\left\langle a_{\mathbf{k}}^{+} W(\mathbf{q}) a_{\mathbf{k}-\mathbf{q}}\right\rangle\right\rangle_{\omega}= \\
= & \left\langle a_{\mathbf{k}}^{+} a_{\mathbf{k}-\mathbf{q}}\right\rangle+\sum_{\mathbf{q}^{\prime}}\left[\left\langle\left\langle a_{\mathbf{k}}^{+} W(\mathbf{q}) W\left(\mathbf{q}^{\prime}\right) a_{\mathbf{k}-\mathbf{q}-\mathbf{q}^{\prime}}\right\rangle\right\rangle_{\omega}-\left\langle\left\langle a_{\mathbf{k}+\mathbf{q}^{\prime}}^{+} W\left(\mathbf{q}^{\prime}\right) W(\mathbf{q}) a_{\mathbf{k}-\mathbf{q}}\right\rangle\right\rangle_{\omega}\right] \\
& +\sum_{n}\left[\left\langle\left\langle a_{\mathbf{k}}^{+} W(\mathbf{q}) \Delta_{\mathbf{k}-\mathbf{q}, n} b_{n}\right\rangle\right\rangle_{\omega}-\left\langle\left\langle b_{n}^{+} \Delta_{n \mathbf{k}} W(\mathbf{q}) a_{\mathbf{k}-\mathbf{q}}\right\rangle\right\rangle_{\omega}\right] \\
\left(\varepsilon_{\mathbf{k}+\mathbf{q}}\right. & \left.-\varepsilon_{\mathbf{k}}+\hbar \omega\right)\left\langle\left\langle a_{\mathbf{k}+\mathbf{q}}^{+} W(\mathbf{q}) a_{\mathbf{k}}\right\rangle\right\rangle_{\omega}= \\
= & \left\langle a_{\mathbf{k}+\mathbf{q}}^{+} a_{\mathbf{k}}\right\rangle+\sum_{\mathbf{q}^{\prime}}\left[\left\langle\left\langle a_{\mathbf{k}+\mathbf{q}}^{+} W(\mathbf{q}) W\left(\mathbf{q}^{\prime}\right) a_{\mathbf{k}-\mathbf{q}}\right\rangle\right\rangle_{\omega}-\left\langle\left\langle a_{\mathbf{k}+\mathbf{q}+\mathbf{q}^{\prime}}^{+} W\left(\mathbf{q}^{\prime}\right) W(\mathbf{q}) a_{\mathbf{k}}\right\rangle\right\rangle_{\omega}\right] \\
& +\sum_{n}\left[\left\langle\left\langle a_{\mathbf{k}+\mathbf{q}}^{+} W(\mathbf{q}) \Delta_{\mathbf{k} n} b_{n}\right\rangle\right\rangle_{\omega}-\left\langle\left\langle b_{n}^{+} \Delta_{n, \mathbf{k}+\mathbf{q}} W(\mathbf{q}) a_{\mathbf{k}}\right\rangle\right\rangle_{\omega}\right] \\
\left(\varepsilon_{\mathbf{k}}-\right. & \left.\varepsilon_{n}+\hbar \omega\right)\left\langle\left\langle a_{\mathbf{k}}^{+} \Delta_{\mathbf{k} n} b_{n}\right\rangle\right\rangle_{\omega}= \\
= & \left\langle a_{\mathbf{k}}^{+} \Delta_{\mathbf{k} n}\right\rangle-\sum_{\mathbf{q}}\left\langle\left\langle a_{\mathbf{k}+\mathbf{q}}^{+} W(\mathbf{q}) \Delta_{\mathbf{k} n} b_{n}\right\rangle\right\rangle_{\omega}+\sum_{\mathbf{k}^{\prime}}\left\langle\left\langle a_{\mathbf{k}}^{+} \Delta_{\mathbf{k} n} \Delta_{n \mathbf{k}^{\prime}} a_{\mathbf{k}^{\prime}}\right\rangle\right\rangle_{\omega} \\
& -\sum_{n^{\prime}}\left\langle\left\langle b_{n^{\prime}}^{+} \Delta_{n^{\prime} \mathbf{k}} \Delta_{\mathbf{k} n} b_{n}\right\rangle\right\rangle_{\omega}, \\
\left(\varepsilon_{n}-\right. & \left.\varepsilon_{\mathbf{k}}+\hbar \omega\right)\left\langle\left\langle b_{n}^{+} a_{\mathbf{k}} \Delta_{n \mathbf{k}}\right\rangle\right\rangle_{\omega}= \\
= & \left\langle b_{n}^{+} \Delta_{n \mathbf{k}}\right\rangle+\sum_{\mathbf{q}}\left\langle\left\langle b_{n}^{+} \Delta_{n \mathbf{k}} W(\mathbf{q}) a_{\mathbf{k}-\mathbf{q}}\right\rangle\right\rangle_{\omega}+\sum_{n^{\prime}}\left\langle\left\langle b_{n}^{+} \Delta_{n \mathbf{k}} \Delta_{\mathbf{k} n^{\prime}} b_{n^{\prime}}\right\rangle\right\rangle_{\omega} \\
& -\sum_{\mathbf{k}^{\prime}}\left\langle\left\langle a_{\mathbf{k}^{\prime}}^{+} \Delta_{\mathbf{k}^{\prime} n} \Delta_{n \mathbf{k}} a_{\mathbf{k}}\right\rangle\right\rangle_{\omega} .
\end{aligned}
$$

\section{Second order perturbation theory}

Certain sums in the right parts of the last four equations contain an item of the value comparable to the contribution of the rest summands. These items are the Green functions containing weakly coupled averages. If we take into account only 
these and neglect all the other summands we will find the conductivity in the lowest (quadratic) order of perturbation theory by hybridization and by pseudo-potential. Considering only the weakly coupled averages we can write down the system:

$$
\begin{aligned}
& \left(\varepsilon_{\mathbf{k}}-\varepsilon_{\mathbf{k}-\mathbf{q}}+\hbar \omega\right)\left\langle\left\langle a_{\mathbf{k}}^{+} W(\mathbf{q}) a_{\mathbf{k}-\mathbf{q}}\right\rangle\right\rangle_{\omega}= \\
& \quad=\left\langle\left\langle a_{\mathbf{k}}^{+} W(\mathbf{q}) W\left(\mathbf{q}^{\prime}\right) a_{\mathbf{k}}\right\rangle\right\rangle_{\omega}-\left\langle\left\langle a_{\mathbf{k}-\mathbf{q}}^{+} W(-\mathbf{q}) W(\mathbf{q}) a_{\mathbf{k}-\mathbf{q}}\right\rangle\right\rangle_{\omega}, \\
& \left(\varepsilon_{\mathbf{k}+\mathbf{q}}-\varepsilon_{\mathbf{k}}+\hbar \omega\right)\left\langle\left\langle a_{\mathbf{k}+\mathbf{q}}^{+} W(\mathbf{q}) a_{\mathbf{k}}\right\rangle\right\rangle_{\omega}= \\
& \quad=\left\langle\left\langle a_{\mathbf{k}+\mathbf{q}}^{+} W(\mathbf{q}) W\left(\mathbf{q}^{\prime}\right) a_{\mathbf{k}+\mathbf{q}}\right\rangle\right\rangle_{\omega}-\left\langle\left\langle a_{\mathbf{k}}^{+} W(-\mathbf{q}) W(\mathbf{q}) a_{\mathbf{k}}\right\rangle\right\rangle_{\omega} \\
& \left(\varepsilon_{\mathbf{k}}-\varepsilon_{n}+\hbar \omega\right)\left\langle\left\langle a_{\mathbf{k}}^{+} \Delta_{\mathbf{k} n} b_{n}\right\rangle\right\rangle_{\omega}=\left\langle\left\langle a_{\mathbf{k}}^{+} \Delta_{\mathbf{k} n} \Delta_{n \mathbf{k}} a_{\mathbf{k}}\right\rangle\right\rangle_{\omega} \\
& \left(\varepsilon_{n}-\varepsilon_{\mathbf{k}}+\hbar \omega\right)\left\langle\left\langle b_{n}^{+} \Delta_{n \mathbf{k}} a_{\mathbf{k}}\right\rangle\right\rangle_{\omega}=-\left\langle\left\langle a_{\mathbf{k}}^{+} \Delta_{\mathbf{k} n} \Delta_{n \mathbf{k}} a_{\mathbf{k}}\right\rangle\right\rangle_{\omega} .
\end{aligned}
$$

At that, in the lowest order of perturbation theory we have neglected the absolute terms of equations. From the formal point of view this is plausible in the calculations of the terms up to the 4 -th order.

To derive the closed set of equations for the needed Green function we must uncouple all the functions that differ from the sought one. Our approach consists in the assumption that only the functions containing weakly coupled averages should be uncoupled, namely

$$
\begin{aligned}
\left\langle\left\langle a_{\mathbf{k}}^{+} W(\mathbf{q}) W(-\mathbf{q}) a_{\mathbf{k}}\right\rangle\right\rangle_{\omega} & =\langle W(\mathbf{q}) W(-\mathbf{q})\rangle\left\langle\left\langle a_{\mathbf{k}}^{+} a_{\mathbf{k}}\right\rangle\right\rangle_{\omega} \\
\left\langle\left\langle a_{\mathbf{k}}^{+} \Delta_{\mathbf{k} n} \Delta_{n \mathbf{k}} a_{\mathbf{k}}\right\rangle\right\rangle_{\omega} & =\left\langle\Delta_{\mathbf{k} n} \Delta_{n \mathbf{k}}\right\rangle\left\langle\left\langle a_{\mathbf{k}}^{+} a_{\mathbf{k}}\right\rangle\right\rangle_{\omega} .
\end{aligned}
$$

Uncoupling of this type is widely used in various fields of nowadays theoretical physics and is usually referred to as the mean field approximation. In the particular case of electron transport phenomena it means that we consider the scattering of the electrons on the averaged potential produced by the whole ion subsystem, instead of examining the scattering on the specific ion. The precision of this approximation increases as the mean free path of the conductance electron grows. Finally, we can rewrite the first five equations of the set in the form of a single equation for the wanted Green function. In the static case this equation looks as follows:

$$
\begin{aligned}
&\left\langle a_{\mathbf{k}}^{+}(0) a_{\mathbf{k}}(0)\right\rangle= \mathrm{i} \frac{2 \pi N}{V^{2}} \sum_{\mathbf{q}} w^{2}(q) S(q) \delta\left(\varepsilon_{\mathbf{k}}-\varepsilon_{\mathbf{k}+\mathbf{q}}\right) \\
& \quad \times\left[\left\langle\left\langle a_{\mathbf{k}}^{+}(t) a_{\mathbf{k}}(t)\right\rangle\right\rangle_{0}-\left\langle\left\langle a_{\mathbf{k}+\mathbf{q}}^{+}(t) a_{\mathbf{k}+\mathbf{q}}(t)\right\rangle\right\rangle_{0}\right] \\
&+\mathrm{i} \frac{2 \pi}{V} \sum_{n} \Delta_{k}^{2} \delta\left(\varepsilon_{\mathbf{k}}-\varepsilon_{n}\right)\left\langle\left\langle a_{\mathbf{k}}^{+}(t) a_{\mathbf{k}}(t)\right\rangle\right\rangle_{0} .
\end{aligned}
$$

Here we have used the well-known operator relation

$$
\frac{1}{x+\mathrm{i} \delta}=P \frac{1}{x}-\mathrm{i} \pi \delta(x)
$$

and have introduced the static structure factor of ion subsystem

$$
S(q)=\frac{1}{N}\left\langle\rho^{i}(\mathbf{q}) \rho^{i}(-\mathbf{q})\right\rangle .
$$


The kinetic equation derived differs from the one for the simple metals by the last term only. As well as its exact solution is quite analogous and based on the result precise to a second order of perturbation theory

$$
\left\langle\left\langle a_{\mathbf{k}+\mathbf{q}}^{+}(t) a_{\mathbf{k}+\mathbf{q}}(t)\right\rangle\right\rangle_{0}=\frac{\mathbf{k}+\mathbf{q}}{|\mathbf{k}+\mathbf{q}|}\left\langle\left\langle a_{\mathbf{k}}^{+}(t) a_{\mathbf{k}}(t)\right\rangle\right\rangle_{0}
$$

(see e.g. [1]).

As a result, the solution takes the form

$$
\left\langle\left\langle a_{\mathbf{k}}^{+}(t) a_{\mathbf{k}}(t)\right\rangle\right\rangle_{0}=-\frac{\mathrm{i}}{\hbar}\left\langle a_{\mathbf{k}}^{+}(0) a_{\mathbf{k}}(0)\right\rangle \tau(k)
$$

where

$$
\begin{aligned}
\tau^{-1}(k)= & \frac{2 \pi N}{\hbar V^{2}} \sum_{\mathbf{q}} w^{2}(q) S(q) \delta\left(\varepsilon_{\mathbf{k}}-\varepsilon_{\mathbf{k}+\mathbf{q}}\right)\left[1-\cos \left(\mathbf{k}^{\wedge} \mathbf{k}+\mathbf{q}\right)\right] \\
& +\frac{2 \pi}{\hbar V} \sum_{n} \Delta_{k}^{2} \delta\left(\varepsilon_{\mathbf{k}}-\varepsilon_{n}\right) .
\end{aligned}
$$

is the inverse relaxation time for the conductivity of transition metals. If in the last expression we put $\Delta_{k} \rightarrow 0$, we are left with a well-known Ziman formula for simple metals [1]. After some simplification it can be brought to the form

$$
\tau^{-1}(k)=\frac{N m}{4 \pi V \hbar^{3} k^{3}} \int_{0}^{2 k} \mathrm{~d} x x^{3} w^{2}(x) S(x) .
$$

On the other hand, if we tend $w \rightarrow 0$, our result comes to Mott formula [6]. After the transformation consisting in switching from summation over the quantum numbers to the integration over the energy we will derive

$$
\tau^{-1}(k)=\frac{2 \pi}{\hbar} \Delta_{k}^{2} G\left(\varepsilon_{k}\right)
$$

where $G(\varepsilon)$ is the density of $d$-states.

\section{Third order of perturbation theory}

To do calculations of the terms of the third order we should supply the previously derived set with the following equations:

$$
\begin{aligned}
\left(\varepsilon_{\mathbf{k}}-\right. & \left.\varepsilon_{\mathbf{k}-\mathbf{q}-\mathbf{q}^{\prime}}+\hbar \omega\right)\left\langle\left\langle a_{\mathbf{k}}^{+} W(\mathbf{q}) W\left(\mathbf{q}^{\prime}\right) a_{\mathbf{k}-\mathbf{q}_{-} \mathbf{q}^{\prime}}\right\rangle\right\rangle_{\omega}= \\
= & \left\langle\left\langle a_{\mathbf{k}}^{+} W(\mathbf{q}) W\left(\mathbf{q}^{\prime}\right) W\left(-\mathbf{q}-\mathbf{q}^{\prime}\right) a_{\mathbf{k}}\right\rangle\right\rangle_{\omega} \\
& -\left\langle\left\langle a_{\mathbf{k}-\mathbf{q}-\mathbf{q}^{\prime}}^{+} W(\mathbf{q}) W\left(\mathbf{q}^{\prime}\right) W\left(-\mathbf{q}-\mathbf{q}^{\prime}\right) a_{\mathbf{k}-\mathbf{q}-\mathbf{q}^{\prime}}\right\rangle\right\rangle_{\omega} \\
\left(\varepsilon_{\mathbf{k}+\mathbf{q}^{\prime}}\right. & \left.-\varepsilon_{\mathbf{k}-\mathbf{q}}+\hbar \omega\right)\left\langle\left\langle a_{\mathbf{k}+\mathbf{q}^{\prime}}^{+} W\left(\mathbf{q}^{\prime}\right) W(\mathbf{q}) a_{\mathbf{k}-\mathbf{q}}\right\rangle\right\rangle_{\omega}= \\
= & \left\langle\left\langle a_{\mathbf{k}+\mathbf{q}^{\prime}}^{+} W\left(\mathbf{q}^{\prime}\right) W(\mathbf{q}) W\left(-\mathbf{q}-\mathbf{q}^{\prime}\right) a_{\mathbf{k}+\mathbf{q}^{\prime}}\right\rangle\right\rangle_{\omega}
\end{aligned}
$$




$$
\begin{aligned}
& -\left\langle\left\langle a_{\mathbf{k}-\mathbf{q}}^{+} W\left(-\mathbf{q}-\mathbf{q}^{\prime}\right) W\left(\mathbf{q}^{\prime}\right) W(\mathbf{q}) a_{\mathbf{k}-\mathbf{q}}\right\rangle\right\rangle_{\omega}, \\
\left(\varepsilon_{\mathbf{k}}-\right. & \left.\varepsilon_{n}+\hbar \omega\right)\left\langle\left\langle a_{\mathbf{k}}^{+} W(\mathbf{q}) \Delta_{\mathbf{k}-\mathbf{q}, n} b_{n}\right\rangle\right\rangle_{\omega}= \\
= & \left\langle\left\langle a_{\mathbf{k}}^{+} W(\mathbf{q}) \Delta_{\mathbf{k}-\mathbf{q}, n} \Delta_{n \mathbf{k}} a_{k}\right\rangle\right\rangle_{\omega}, \\
\left(\varepsilon_{n}-\right. & \left.\varepsilon_{\mathbf{k}-\mathbf{q}}+\hbar \omega\right)\left\langle\left\langle b_{n}^{+} \Delta_{n \mathbf{k}} W(\mathbf{q}) a_{\mathbf{k}-\mathbf{q}}\right\rangle\right\rangle_{\omega}= \\
= & -\left\langle\left\langle a_{\mathbf{k}-\mathbf{q}}^{+} \Delta_{\mathbf{k}-\mathbf{q}, n} \Delta_{n \mathbf{k}} W(\mathbf{q}) a_{\mathbf{k}-\mathbf{q}}\right\rangle\right\rangle_{\omega}, \\
\left(\varepsilon_{\mathbf{k}+\mathbf{q}}\right. & \left.-\varepsilon_{\mathbf{k}-\mathbf{q}^{\prime}}+\hbar \omega\right)\left\langle\left\langle a_{\mathbf{k}+\mathbf{q}}^{+} W(\mathbf{q}) W\left(\mathbf{q}^{\prime}\right) a_{\mathbf{k}-\mathbf{q}^{\prime}}\right\rangle\right\rangle_{\omega}= \\
= & \left\langle\left\langle a_{\mathbf{k}+\mathbf{q}}^{+} W(q) W\left(\mathbf{q}^{\prime}\right) W\left(-\mathbf{q}-\mathbf{q}^{\prime}\right) a_{\mathbf{k}+\mathbf{q}}\right\rangle\right\rangle_{\omega} \\
& -\left\langle\left\langle a_{\mathbf{k}-\mathbf{q}^{\prime}}^{+} W\left(-\mathbf{q}-\mathbf{q}^{\prime}\right) W(\mathbf{q}) W\left(\mathbf{q}^{\prime}\right) a_{k-\mathbf{q}^{\prime}}\right\rangle\right\rangle_{\omega}, \\
\left(\varepsilon_{\mathbf{k}+\mathbf{q}+\mathbf{q}^{\prime}}-\varepsilon_{k}+\hbar \omega\right)\left\langle\left\langle a_{\mathbf{k}+\mathbf{q}+\mathbf{q}^{+}} W\left(\mathbf{q}^{\prime}\right) W(\mathbf{q}) a_{\mathbf{k}}\right\rangle\right\rangle_{\omega}= & = \\
= & \left\langle\left\langle a_{\mathbf{k}+\mathbf{q}+\mathbf{q}^{\prime}}^{+} W\left(\mathbf{q}^{\prime}\right) W(\mathbf{q}) W\left(-\mathbf{q}-\mathbf{q}^{\prime}\right) a_{\mathbf{k}+\mathbf{q}+\mathbf{q}^{\prime}}\right\rangle\right\rangle_{\omega} \\
& -\left\langle\left\langle a_{\mathbf{k}}^{+} W\left(-\mathbf{q}-\mathbf{q}^{\prime}\right) W\left(\mathbf{q}^{\prime}\right) W(\mathbf{q}) a_{\mathbf{k}}\right\rangle\right\rangle_{\omega}, \\
\left(\varepsilon_{\mathbf{k}+\mathbf{q}}\right. & \left.-\varepsilon_{n}+\hbar \omega\right)\left\langle\left\langle a_{\mathbf{k}+\mathbf{q}}^{+} W(\mathbf{q}) \Delta_{\mathbf{k} n} b_{n}\right\rangle\right\rangle_{\omega}= \\
= & \left\langle\left\langle a_{\mathbf{k}+\mathbf{q}}^{+} W(\mathbf{q}) \Delta_{\mathbf{k} n} \Delta_{n, \mathbf{k}+\mathbf{q}} a_{\mathbf{k}+\mathbf{q}}\right\rangle\right\rangle_{\omega}, \\
\left(\varepsilon_{n}-\right. & \left.\varepsilon_{\mathbf{k}}+\hbar \omega\right)\left\langle\left\langle b_{n}^{+} \Delta_{n, \mathbf{k}+\mathbf{q}} W(\mathbf{q}) a_{\mathbf{k}}\right\rangle\right\rangle_{\omega}= \\
= & -\left\langle\left\langle a_{\mathbf{k}}^{+} \Delta_{\mathbf{k} n} \Delta_{n, \mathbf{k}+\mathbf{q}} W(\mathbf{q}) a_{\mathbf{k}}\right\rangle\right\rangle_{\omega}, \\
\left(\varepsilon_{\mathbf{k}+\mathbf{q}}\right. & \left.-\varepsilon_{n}+\hbar \omega\right)\left\langle\left\langle a_{\mathbf{k}+\mathbf{q}}^{+} W(\mathbf{q}) \Delta_{\mathbf{k} n} b_{n}\right\rangle\right\rangle_{\omega}= \\
= & \left\langle\left\langle a_{\mathbf{k}+\mathbf{q}}^{+} W(\mathbf{q}) \Delta_{\mathbf{k} n} \Delta_{n, \mathbf{k}+\mathbf{q}} a_{\mathbf{k}+\mathbf{q}}\right\rangle\right\rangle_{\omega}, \\
\left(\varepsilon_{\mathbf{k}}-\right. & \left.\varepsilon_{\mathbf{k}-\mathbf{q}}+\hbar \omega\right)\left\langle\left\langle a_{\mathbf{k}}^{+} \Delta_{\mathbf{k} n} \Delta_{n, \mathbf{k}-\mathbf{q}} a_{\mathbf{k}-\mathbf{q}}\right\rangle\right\rangle_{\omega}= \\
= & \left\langle\left\langle a_{\mathbf{k}}^{+} \Delta_{\mathbf{k} n} \Delta_{n \mathbf{k}} W(-\mathbf{q}) a_{\mathbf{k}}\right\rangle\right\rangle_{\omega} \\
& -\left\langle\left\langle a_{\mathbf{k}-\mathbf{q}}^{+} W(-\mathbf{q}) \Delta_{\mathbf{k} n} \Delta_{n, \mathbf{k}-\mathbf{q}} a_{\mathbf{k}-\mathbf{q}}\right\rangle\right\rangle_{\omega}, \\
\left(\varepsilon_{n}-\right. & \left.\varepsilon_{\mathbf{k}-\mathbf{q}}+\hbar \omega\right)\left\langle\left\langle b_{n}^{+} \Delta_{n \mathbf{k}} W(\mathbf{q}) a_{\mathbf{k}-\mathbf{q}}\right\rangle\right\rangle_{\omega}= \\
= & -\left\langle\left\langle a_{\mathbf{k}-\mathbf{q}}^{+} \Delta_{\mathbf{k}-\mathbf{q}, n} \Delta_{n \mathbf{k}} W(\mathbf{q}) a_{\mathbf{k}-\mathbf{q}}\right\rangle\right\rangle_{\omega}, \\
\left(\varepsilon_{\mathbf{k}+\mathbf{q}}-\right. & \left.-\varepsilon_{\mathbf{k}}+\hbar \omega\right)\left\langle\left\langle a_{\mathbf{k}+\mathbf{q}}^{+} \Delta_{\mathbf{k}+\mathbf{q}, n} \Delta_{n \mathbf{k}} a_{\mathbf{k}}\right\rangle\right\rangle_{\omega}= \\
= & \left\langle\left\langle a_{\mathbf{k}+\mathbf{q}}^{+} \Delta_{\mathbf{k}+\mathbf{q}, n} \Delta_{n \mathbf{k}} W(-\mathbf{q}) a_{\mathbf{k}+\mathbf{q}}\right\rangle\right\rangle_{\omega} \\
& -\left\langle\left\langle a_{\mathbf{k}}^{+} W(-\mathbf{q}) \Delta_{\mathbf{k}+\mathbf{q}, n} \Delta_{n \mathbf{k}} a_{\mathbf{k}}\right\rangle\right\rangle_{\omega} . \\
= &
\end{aligned}
$$

Examining the structure of these equations one can see that the kinetic equation will be widened with terms of the third order by pseudopotential as well as with the terms of the second order by hybridization potential together with the first one by pseudopotential. Here we will discuss the latter only as far as the former is well described in the works devoted to simple metals $[12,13]$. In view of the preceding, the kinetic equation will take the form

$$
\begin{aligned}
\hbar \omega\left\langle\left\langle a_{\mathbf{k}}^{+} a_{\mathbf{k}}\right\rangle\right\rangle_{\omega}= & \left\langle a_{\mathbf{k}}^{+} a_{\mathbf{k}}\right\rangle+\hat{T}_{w w}\left\langle\left\langle a_{\mathbf{k}}^{+} a_{\mathbf{k}}\right\rangle\right\rangle_{\omega}+\hat{T}_{\Delta \Delta}\left\langle\left\langle a_{\mathbf{k}}^{+} a_{\mathbf{k}}\right\rangle\right\rangle_{\omega} \\
& +\hat{T}_{w w w}\left\langle\left\langle a_{\mathbf{k}}^{+} a_{\mathbf{k}}\right\rangle\right\rangle_{\omega}+\hat{T}_{\Delta \Delta w}\left\langle\left\langle a_{\mathbf{k}}^{+} a_{\mathbf{k}}\right\rangle\right\rangle_{\omega} .
\end{aligned}
$$

After the weakly coupled averages are uncoupled, we derive

$$
\hat{T}_{\Delta \Delta w}\left\langle\left\langle a_{\mathbf{k}}^{+} a_{\mathbf{k}}\right\rangle\right\rangle_{\omega}=\sum_{\mathbf{q}, n}\left\langle W(\mathbf{q}) \Delta_{\mathbf{k}+\mathbf{q}, n} \Delta_{n \mathbf{k}}\right\rangle \times
$$




$$
\begin{aligned}
\times & \left\{2\left[\frac{1}{\varepsilon_{\mathbf{k}}-\varepsilon_{\mathbf{k}+\mathbf{q}}+\hbar \omega} \cdot \frac{1}{\varepsilon_{\mathbf{k}}-\varepsilon_{n}+\hbar \omega}-\frac{1}{\varepsilon_{\mathbf{k}+\mathbf{q}}-\varepsilon_{\mathbf{k}}+\hbar \omega} \cdot \frac{1}{\varepsilon_{n}-\varepsilon_{\mathbf{k}}+\hbar \omega}\right]\right. \\
\times & \left\langle\left\langle a_{\mathbf{k}}^{+} a_{\mathbf{k}}\right\rangle\right\rangle_{\omega} \\
+ & {\left[\frac{1}{\varepsilon_{\mathbf{k}}-\varepsilon_{\mathbf{k}+\mathbf{q}}+\hbar \omega} \cdot \frac{1}{\varepsilon_{n}-\varepsilon_{\mathbf{k}}+\hbar \omega}-\frac{1}{\varepsilon_{\mathbf{k}+\mathbf{q}}-\varepsilon_{\mathbf{k}}+\hbar \omega} \cdot \frac{1}{\varepsilon_{\mathbf{k}+\mathbf{q}}-\varepsilon_{n}+\hbar \omega}\right.} \\
& +\frac{1}{\varepsilon_{n}-\varepsilon_{\mathbf{k}}+\hbar \omega} \cdot \frac{1}{\varepsilon_{n}-\varepsilon_{\mathbf{k}+\mathbf{q}}+\hbar \omega}-\frac{1}{\varepsilon_{\mathbf{k}}-\varepsilon_{n}+\hbar \omega} \cdot \frac{1}{\varepsilon_{\mathbf{k}+\mathbf{q}}-\varepsilon_{n}+\hbar \omega} \\
& \left.+\frac{1}{\varepsilon_{n}-\varepsilon_{\mathbf{k}}+\hbar \omega} \cdot \frac{1}{\varepsilon_{\mathbf{k}+\mathbf{q}}-\varepsilon_{\mathbf{k}}+\hbar \omega}-\frac{1}{\varepsilon_{\mathbf{k}}-\varepsilon_{n}+\hbar \omega} \cdot \frac{1}{\varepsilon_{\mathbf{k}}-\varepsilon_{\mathbf{k}+\mathbf{q}}+\hbar \omega}\right] \\
\times & \left.\left\langle\left\langle a_{\mathbf{k}+\mathbf{q}}^{+} a_{\mathbf{k}+\mathbf{q}}\right\rangle\right\rangle_{\omega}\right\} .
\end{aligned}
$$

Using the well-known operator identity in a static case $\omega=0$ we can write the term under consideration as

$$
\begin{aligned}
& \hat{T}_{\Delta \Delta w}\left\langle\left\langle a_{\mathbf{k}}^{+} a_{\mathbf{k}}\right\rangle\right\rangle_{0}=-4 \pi \mathrm{i} \sum_{q, n}\left\langle W(-\mathbf{q}) \Delta_{\mathbf{k}+\mathbf{q}, n} \Delta_{n \mathbf{k}}\right\rangle \\
& \quad \times\left\{\frac{\delta\left(\varepsilon_{\mathbf{k}}-\varepsilon_{\mathbf{k}+\mathbf{q}}\right)}{\varepsilon_{\mathbf{k}}-\varepsilon_{n}}\left[\left\langle\left\langle a_{\mathbf{k}}^{+} a_{\mathbf{k}}\right\rangle\right\rangle_{0}-\left\langle\left\langle a_{\mathbf{k}+\mathbf{q}}^{+} a_{\mathbf{k}+\mathbf{q}}\right\rangle\right\rangle_{0}\right]+\frac{\delta\left(\varepsilon_{\mathbf{k}}-\varepsilon_{n}\right)}{\varepsilon_{\mathbf{k}}-\varepsilon_{\mathbf{k}+\mathbf{q}}}\left\langle\left\langle a_{\mathbf{k}}^{+} a_{\mathbf{k}}\right\rangle\right\rangle_{0}\right\} .
\end{aligned}
$$

Let us do a thorough analysis of the average under summation

$$
\left\langle W(\mathbf{q}) \Delta_{\mathbf{k}+\mathbf{q}, n} \Delta_{n \mathbf{k}}\right\rangle=\frac{1}{V^{2}} w(q) \Delta_{k} \Delta_{|\mathbf{k}+\mathbf{q}|}\left\langle\rho^{i}(-\mathbf{q}) \exp \left(-\mathrm{iq} \mathbf{R}_{n}\right)\right\rangle
$$

It is helpful to divide the summation over the " $\mathrm{n}$ " into two sums. The first of them corresponds to the summation over the set of quantum numbers, determining the electron energy. The second one corresponds to the summation over the ion positions. Taking the assumption that the electron energy $\varepsilon_{n}$ doesn't depend on the position of a specific ion we can do a significant simplification. The potential of hybridization depends in turn on the ion positions only. In that case we can easily carry out the summation and express the result

$$
\sum_{n}\left\langle W(\mathbf{q}) \Delta_{\mathbf{k}+\mathbf{q}, n} \Delta_{n \mathbf{k}}\right\rangle=\frac{N}{V^{2}} w(q) \Delta_{k} \Delta_{|\mathbf{k}+\mathbf{q}|} S(q)
$$

via the static structure factor of the ion subsystem. There is only a summation over the electron states in the ion left in the kinetic equation. As far as the formfactor of hybridization potential depends purely on the absolute value of wave vector, the only multiplier in the integral equation kernel that depends on the angle between $\mathbf{k}$ and $\mathbf{k}+\mathbf{q}$ is delta function. On the whole, the structure of the third order term under consideration is equivalent to the structure of the second order term as the angle dependence is regarded. So, we can put the third order term in a similar way

$$
\hat{T}_{\Delta \Delta w}\left\langle\left\langle a_{\mathbf{k}}^{+} a_{\mathbf{k}}\right\rangle\right\rangle_{0}=-\mathrm{i} \hbar \tau_{\Delta \Delta w}^{-1}(k)\left\langle\left\langle a_{\mathbf{k}}^{+} a_{\mathbf{k}}\right\rangle\right\rangle_{0},
$$


where the contribution of the third order term to the inverse relaxation time has the form

$$
\begin{aligned}
\hat{T}_{\Delta \Delta w}(k)= & -\frac{4 \pi N}{\hbar V^{2}} \sum_{\mathbf{q}, n} w(q) \Delta_{k} \Delta_{|\mathbf{k}+\mathbf{q}|} S(q) \\
& \times\left\{\frac{\delta\left(\varepsilon_{\mathbf{k}}-\varepsilon_{n}\right)}{\varepsilon_{\mathbf{k}}-\varepsilon_{\mathbf{k}+\mathbf{q}}}+\frac{\delta\left(\varepsilon_{\mathbf{k}}-\varepsilon_{\mathbf{k}+\mathbf{q}}\right)}{\varepsilon_{\mathbf{k}}-\varepsilon_{n}}\left[1-\cos \left(\mathbf{k}^{\wedge} \mathbf{k}+\mathbf{q}\right)\right]\right\} .
\end{aligned}
$$

Usually, when the summation over the electron energy states in all the ions is performed, one can allow for a disorder of the ion system introducing the energy distribution function, namely

$$
\sum_{n} \rightarrow \int \mathrm{d} \varepsilon G(\varepsilon)
$$

It is assumed that the function $G(\varepsilon)$ is equal to electron density states up to normalization factor. In our case it is $d$-states. So that

$$
\begin{aligned}
\tau_{\Delta \Delta w}^{-1}(k)= & \frac{4 \pi N}{\hbar V^{2}} \sum_{\mathbf{k}^{\prime}} w\left(\left|\mathbf{k}-\mathbf{k}^{\prime}\right|\right) \Delta_{k} \Delta_{k^{\prime}} S\left(\left|\mathbf{k}-\mathbf{k}^{\prime}\right|\right) \\
& \times\left\{\frac{G\left(\varepsilon_{k}\right)}{\varepsilon_{\mathbf{k}}-\varepsilon_{\mathbf{k}^{\prime}}}+\left[1-\cos \left(\mathbf{k}^{\wedge} \mathbf{k}^{\prime}\right)\right] \delta\left(\varepsilon_{\mathbf{k}}-\varepsilon_{\mathbf{k}^{\prime}}\right) \int_{-\infty}^{\infty} \frac{G(\varepsilon)}{\varepsilon_{\mathbf{k}}-\varepsilon} \mathrm{d} \varepsilon\right\} .
\end{aligned}
$$

To derive the final result we should replace the summation over the wave vector by integration. In a traditional approach

$$
\begin{aligned}
\tau_{\Delta \Delta w}^{-1}\left(k_{\mathrm{F}}\right)= & \frac{1}{6 \pi^{3} z} \Delta_{k_{\mathrm{F}}}^{2} \int_{0}^{2 k_{\mathrm{F}}} w(x) S(x) x^{3} \mathrm{~d} x \int_{-\infty}^{\infty} \frac{G(\varepsilon)}{\varepsilon_{\mathrm{F}}-\varepsilon} \mathrm{d} \varepsilon \\
& +\frac{2 k_{\mathrm{F}}^{2}}{3 \pi^{3} z} \Delta_{k_{\mathrm{F}}} G\left(\varepsilon_{\mathrm{F}}\right) \int_{0}^{\infty} \mathrm{d} k k \frac{\Delta_{k}}{k_{\mathrm{F}}^{2}-k^{2}} \int_{\left|k_{\mathrm{F}}-k\right|}^{k_{\mathrm{F}}+k} w(x) S(x) x \mathrm{~d} x
\end{aligned}
$$

where $z$ is the valence of ion. The electrical resistance can be found using a wellknown formula of Lorenz at that.

\section{Discussion}

If within the framework of Mott model we suppose that the only considerable potential determining the electron scattering is the hybridization potential, then the third order term in the expansion of the inverse relaxation time will disappear. It is easy to see if we tend pseudopotential to zero. The term following the quadratic will be of the fourth order in this approach.

First of all, we should notice that the derived result generalizes the Mott formula yet in the second order of perturbation theory. The importance of the terms of expansion by pseudopotential is clarified by the following estimation. Let us, for example, put the disordered $3 d$-metals under consideration. This row in periodic 
table is closed by the $\mathrm{Cu}$, that has completed $3 d$-states. An accurate calculation of its resistance is possible only while taking into account the pseudopotential, and results approximately in $20 \mu \Omega \cdot \mathrm{cm}$. For all the other metals in the row, it varies from $80 \mu \Omega \cdot \mathrm{cm}$ to $400 \mu \Omega \cdot \mathrm{cm}$. Considering the pseudopotential contribution to be equal for all $3 d$-metals it amounts from 5 to 25 percent from the total value. The estimation of the third order term can be done in the same approach. To do this, we need to multiply the second order hybridization term by a square root of the second order pseudopotential term. The resulting range is $20-50 \%$.

There is a qualitative conclusion that can be drawn from the result obtained. It is known that the electrical resistance decreases as the number of $d$-electrons grows. Our correction contains two components. The sign of the first one doesn't depend on mutual disposal of the Fermi level and the middle of $d$-zone. It is completely determined by the behaviour of hybridization potential formfactor in the entire region of integration. On the contrary, the sign of the second component depends on the mutual disposal of the energy levels. As long as

$$
\int_{-\infty}^{\infty} \frac{G(\varepsilon)}{\varepsilon_{\mathrm{F}}-\varepsilon} \mathrm{d} \varepsilon>0, \quad \varepsilon_{\mathrm{F}}>\varepsilon_{0}
$$

and

$$
\int_{-\infty}^{\infty} \frac{G(\varepsilon)}{\varepsilon_{\mathrm{F}}-\varepsilon} \mathrm{d} \varepsilon<0, \quad \varepsilon_{\mathrm{F}}<\varepsilon_{0} .
$$

Usually we have

$$
\int_{0}^{2 k_{\mathrm{F}}} W(x) S(x) x^{3} \mathrm{~d} x<0,
$$

so, the second component will be positive for the metals with a few $d$-electrons (less than 5) and negative for those with a large number of $d$-electrons (more than 5). Correspondingly, the electrical resistance is higher in the first case.

\section{References}

1. Ziman J.M.A. A theory of properties of liquid metals. // Phil. Mag., 1961, vol. 6, No. 68, p. 1013-1034.

2. Shvets V.T. The choice of the pseudopotential and the electrical resistivity of simple disordered metals. // The physics of metals and metallography, 2000, vol. 89, No. 3, p. 211-216.

3. Evans R., Greenwood P.A., Zloyd P. Calculations of the transport properties of liquid transition metals. // Phys. Lett. A., 1971, vol. 35, No. 2, p. 57-58.

4. Hirata K., Waseda Y., Jain A., Srivastava R. Resistivity of liquid transition metals and their alloys using the $t$ matrix. // J. Phys. F: Metal Phys., 1977, vol. 7, No. 3, p. $419-425$.

5. Danleavy H.N., Jones W. Multiple scattering calculations of the resistivity of liquid transition metals. // J. Phys. F: Metal Phys., 1978, vol. 8, No. 7, p. 1477-1482. 
6. Mott N.F. The electrical resistivity of liquid transition metals. // Phil. Mag., 1972, vol. 26, No. 1, p. 1249-1261.

7. Hiroshi T. Scheme for ab initio calculations of the Green function in large disordered systems with application to transport properties. // Phys. Rev. B., 1998, vol. 57, No. 4, p. 2168-2173.

8. Hiroshi T., Masaki I. Ab initio calculation of the Hall conductivity: positive Hall coefficient of liquid Fe. // Phys. Rev. Lett., 1998, vol. 81, No. 17, p. 3727-3730.

9. Masaki I, Hiroshi T. Diamagnetic current and positive Hall coefficients of disordered metals. // J. Phys.: Condens. Matter, 1998, vol. 10, p. 7383-7390.

10. Shvets V.T. Positive Hall's constant for transition disordered metals. // Ukr. J. Phys., 1998, vol. 43, No. 1, p. 45-54.

11. Shvets V.T. Many-partical theory of electron transport processes in transition liquid and amorphous metals. // Materials Science and Engineering B, 1994, vol. 26, p. 141145.

12. Shvets V.T. Temperature electroconductivity coefficient of simple liquid metals. // High Temperature Physics, 2001, vol. 39, No. 1, p. 53-57.

13. Shvets V.T. The comparative analysis of the perturbations theory for electroconductivity of simple disordered metals. // Metal Physics and Advanced Technologies, 2001, vol. 23 , No. 6 , p. $745-753$. 


\title{
Теорія збурень для електроопору перехідних рідких
} металів

\author{
В.Швець, С.Савенко, С.Дацько \\ Одеська державна академія холоду, \\ 65026 Одеса, вул. Дворянська, 1/3
}

Отримано 10 квітня 2002 р.

\begin{abstract}
Для підсистеми $s$-електронів перехідних рідких металів використане наближення майже вільних електронів. $d$-електрони вважаються зв'язаними і грають роль додаткових факторів розсіювання. Модельний гамільтоніан електронної підсистеми містить два малих параметри: псевдопотенціал взаємодії $s$-електронів з іонами і потенціал гібридизації $s$ - $\mathrm{i} d$-електронів. Для знаходження коефіцієнта електропровідності використовується теорія лінійної реакції Кубо і метод двочасових загаяних функцій Гріна. Для електроопору перехідних рідких металів вперше отримано ряд теорії збурень за двома малими параметрами теорії. Електрон-електронна взаємодія врахована у наближенні випадкових фаз. Детально проаналізовані члени другого і третього порядків теорії збурень. Оцінена їх роль у формуванні електроопору перехідних рідких металів.
\end{abstract}

Ключові слова: електроопір, метал

PACS: $72.14 . E, 72.15 . C$ 